\title{
Evaluation of Cystatin C as A Predictor of Coronary Artery Disease in Type 2 Diabetes
}

\author{
Bablu Sukla Das ${ }^{\#}$, Premchandra Singh Y*, Tilakeswari L^^,Joshita L ${ }^{\#}$, Romola \\ Th \# \\ Shaini L***,Sachin Deba Th**, M Amuba Singh*^ \\ \# PGT Biochemistry, RIMS Imphal, *ex-PGT Biochemistry,RIMS,MD (Medicine)student,Taxila American \\ University, ${ }^{\wedge}$ Ex-PGT Anarsthesiology, RIMS, **Associate Professor (Cardiology) RIMS., ***Associate Professor \\ (Biochemistry)., *^ Professor and HOD(Biochemistry) RIMS.
}

\begin{abstract}
Introduction: The association between Coronary Artery Disease (CAD) and diabetes is strong and has led to screening strategies in diabetic patients even before they are symptomatic. Diabetic patients often are unaware of myocardial ischemic pain, and so silent myocardial infarction and ischemia are markedly increased in this population. There is a high chance of developing sudden cardiac death in those with diabetes.

Aims and Objects: To estimate the serum total cystatin C (CsTc) value in type 2 diabetes mellitus (DM-2) with $C A D$ and in normal individuals. and to find out the correlation of serum CsTc, Lipid parameters and Glycosylated Hemoglobin (HbAlc) between patients of DM-2 with or without CAD.

Materials And Methods: The study was conducted in Department of Biochemistry, RIMS in collaboration with Dept of Medicine, RIMS, Imphal, Manipur and Dept of Anaesthesiology \&ICU,RIMS,Imphal for a period of two years. Cases: 60 patients above 18 years of age, fulfilling criteria of diagnosis of Type 2 diabetes mellitus based on WHO definition, which were divided into two subgroups of 30 patients each. Dm-Group: DM-2 (controlled or without complications) and Dm+Cad -Group: DM-2 with confirmed cardiovascular complications. Clinical data, Lipid parameters, blood Glucose, $H B A_{i} c$ were estimated by standard methods. Statistical analysis were done

Conclusion: The study have shown a positive and strong evidence of higher mean serum CsTc levels in patients of DM-2 with CAD suggesting that total serum CsTc level is one of the risk factors predisposingCAD. Total serum CsTc levels are found to be higher in male both in patients of DM-2 with and without CAD as well as in controls compared to their respective female counterparts. Thus, a serum CsTc level finds a link an endothelial dysfunction marker and the development of coronary artery disease.

Key Words:Coronary Artery Disease, cystatin C, type 2 diabetes mellitus
\end{abstract}

\section{Introduction}

The association between Coronary Artery Disease (CAD) and diabetes is strong and has led to screening strategies in diabetic patients even before they are symptomatic. Diabetic patients often are unaware of myocardial ischemic pain, and so silent myocardial infarction and ischemia are markedly increased in this population. There is a high chance of developing sudden cardiac death in those with diabetes ${ }^{[1]}$ Decreased kidney function is associated with adverse cardiovascular outcome. Cystatin C (CsTc) is a novel measure of kidney function that appears to have even stronger associations with mortality and cardiovascular risk than creatinine or estimated glomerular filtration rate (GFR) in the ambulatory elderly and in hospitalized acute coronary syndrome (ACS) patients. ${ }^{[2]}$

Tanil H et a $\boldsymbol{l}^{[3]}$ first discovered CsTc in 1961 as an alkaline protein in normal cerebrospinal fluid. It was a $13-\mathrm{kDa}$, non-glycosylated basic protein belonging to the cystatine super-family of cystatin proteinase inhibitors. Whether the increased cardiovascular risk associated with CsTc elevation was due to increased ischemic burden, higher risk of plaque rupture or alternative mechanisms was unknown. The main cause of death in diabetic patients was cardiovascular disease. It has been reported that two out of every three diabetic patients die of heart failure, myocardial infarction and stroke. Loss of kidney function was a major risk factor for cardiovascular disease. ${ }^{[4]}$

The current gold standard for estimation of renal function is the glomerular filtration rate (GFR), but this test is time consuming and expensive, thus precluding its routine use in clinical settings. ${ }^{[5]}$ Globally, diabetes mellitus (DM) is a major threat to human health. The number of people with diabetes has increased alarmingly since 1985. In 1985, as estimated 30 million people worldwide had diabetes; by 2003 , it was estimated that approximately 194 million people had diabetes, and this figure is expected to rise about 350 million by $2025 .{ }^{[6]}$ 
Seliger SL $\boldsymbol{e t} \boldsymbol{a l}{ }^{[7]}$ have prospectively investigated the association of plasma CsTc levels and ACS. The problem of how blood glucose may be implicated in CAD has been approached in three main ways by examining: the role of blood glucose as a risk factor for $\mathrm{CAD}$ in the general population; the relationship

between hyperglycaemia and CAD incidence in type-2 diabetes mellitus (DM-2) subjects; the effect of treating hyperglycaemia on CAD incidence in DM-2 subjects.In human CsTc is seen in all nucleated cells as a chain of 120 amino acids. It is found in virtually all tissues and body fluids. It is produced at a stable rate, which is unaffected by inflammatory processes, sex, age, diet, and nutritional status.

Only a few circumstances have been identified that have an impact on the production of CsTc, such as very large doses of glucocorticoids and thyroid dysfunction. Biological function of human CsTc and its role in various pathological states has been the subject of numerous studies. ${ }^{[8]}$ In the normal kidney, CsTc is freely filtered through the glomerular membrane and then almost completely reabsorbed and degraded by the proximal tubular cells. Therefore, the plasma concentration of CsTc is almost exclusively determined by the GFR, making it an excellent indicator of GFR. ${ }^{[9]}$

Cystatin C as Biomarker :Imbalance between CsTc and cysteine proteinases is associated with diseases such as inflammation, renal failure, cancer, Alzheimer disease, multiple sclerosis \& hereditary CsTc amyloid angiopathy. Its increased level has been found in patients with autoimmune diseases, with colorectal tumors \& metastases, patients with inflammation and in patients on dialysis. ${ }^{[10]}$ Cystatin $\mathrm{C}$ or cystatin 3 (formerly gamma trace, post-gamma-globulin or neuroendocrine basic polypeptide), a protein encoded by the cystatin-3 (CST3) gene, is mainly used as a biomarker of kidney function. ${ }^{[11]}$ Recently, it has been studied for its role in predicting new-onset or deteriorating cardiovascular disease by Kiuchi $\mathbf{J}$ et al. ${ }^{[12]}$

Although, many studies have been done so far in different parts of the globe regarding CsTc as a biomarker for predicting CAD in DM-2, no such studies have been carried out in the north- eastern states of India. Keeping in view of the reported genetic variation in the association and to assess its utility, this study was aimed to investigate the serum level of CsTc in predicting the complication of cardiovascular disease among individuals suffering from CAD with diabetes in the state of Manipur.

\section{Aims And Objects}

i). To estimate the serum total cystatin $\mathrm{C}$ value in type 2 diabetes mellitus with coronary artery disease and in normal individuals. ii). To find out the correlation of serum cystatin C, Lipid parameters and Glycosylated Hemoglobin between patients of type 2 Diabetes Mellitus with or without CAD.

\section{Materials And Methods}

A cross sectional study was carried out in the Department of Biochemistry, Regional Institute of Medical Sciences (RIMS), Imphal in collaboration with Department of Medicine, RIMS, Imphal, Manipur and Dept of Anaesthesiology\&ICU,RIMS Imphal, for a period of two years. Cases: 60 patients above 18 years of age, fulfilling criteria of diagnosis of Type 2 diabetes mellitus based on WHO definition, attending diabetes clinic and or admitted in the Medicine or ICU ward of Regional Institute of Medical Sciences Hospital were selected randomly irrespective of sex and socioeconomic status. They were divided into two subgroups of 30 patients each.Dm-Group: DM-2 (controlled or without complications)and Dm+Cad -Group: DM-2 with confirmed cardiovascular complications. The diagnosis of cardiovascular complications namely a) coronary artery disease viz. myocardial infarction (MI), angina and b) Ischemic heart disease (IHD) was based on history, clinical findings and findings from serial tracing of 12 lead ECG.Control: 30 healthy and age-sex matched subjects, who were otherwise free from any systemic diseases, were included in the control group.

Exclusion Criteria:Patients with history of acute infections, thyroid dysfunctions and confirmed diagnosed cases of congenital or structural diseases, chronic obstructive pulmonary diseases were excluded.

Methods: AllPatients were selected by randomization after informed consent .A detailed clinical history including age, sex, duration of diseases, age of onset of disease, personal history presence of hypertension, associated symptoms like polyuria, chest pain, palpitation were noted down.

Baseline examination and measurement: Systolic and diastolic blood pressures, weight, BMI, waist to hip ratio were measured for each subject. Subjects were examined in light clothing for the weight. BMI was calculated by body weight $(\mathrm{Kg}) / \mathrm{m}^{2}$ of height. Waist circumference was defined as the smallest girth mid-way between the lowest rib margin and iliac crest. Hip circumference was measured at the level of greater trochanter. Waist-Hip ratio was calculated as waist circumference divided by hip circumference. Base line investigations data, ECG, chest X-ray, available with the patient was taken as reference.The study was done after obtaining the approval from Institutional Ethical Committee of RIMS. 
Collection of blood sample: After obtaining proper well informed written consent of the selected patients, fasting venous blood sample $(5 \mathrm{ml})$ was drawn from antecubital vein. $1 \mathrm{ml}$ of blood was collected in the sterile EDTA vial for the estimation of $\mathrm{HbAlc}$ ) and $3 \mathrm{ml}$ of blood in sterile plain vial (vacutainer) for the estimation of serum CsTc, creatinine, lipid parameters and $1 \mathrm{ml}$ of blood was collected in the fluoride vial for fasting blood glucose.The sample collected in the plain vial was centrifuged immediately for estimation of serum CsTc and serum lipid levels. All the tests were carried out on the same day or within the next 24 hours by keeping the serum in the refrigerator.

Chemicals: All the chemical and reagents used in the study were of analytical grades.

Laboratory methods used:Estimation of HbA1c:Glycosylated haemoglobin was estimated by Fast Ion Exchange Resin Separation Method as described by Goldstein DE, Little RR \& Widdmayer HM (1990). ${ }^{[13]}$

Estimation of serum lipids:Serum lipid profile estimation was estimated by standard laboratory methods $^{[14,15,16,17]}$

Estimation of Serum CsTc: Serum Cystatin C was estimated by ELISA ${ }^{[18]}$

Test Principle: Refrigerator stored serum samples, all reagents supplied with the kits were brought to the room temperature. Standards, quality controls and samples were incubated in micro titrate plate wells pre-coated with polyclonal anti-human CsTc antibody. After 30 minutes incubation and washing, polyclonal anti-humanCsTc antibody, conjugated with horseradish peroxidase (HRP) were added to the wells and incubated for 30 minutes with capturedCsTc. Following another washing step, the remaining HRP conjugate were allowed to react with the substrate solution (TMB). The reaction was stopped by addition of acidic solution and absorbance of the resulting yellow product was measured. The absorbance was proportional to the concentration of CsTc. A standard curve was constructed by plotting absorbance values against concentrations of CsTc standards, and concentrations of unknown samples.

Statistical analysis was performed using SPSS (Statistical Package for Social Science) 11.5 version. Appropriate statistical formulae like $\square^{2}$-test, F-test (ANOVA) and correlation technique were used to analyze the data sets. The P-values less than 0.05 and 0.01 were treated as significant and highly significant values respectively.

\section{Results And Observations:}

.Table-1. Group-wise distribution of cases according to Socio - demographic factors:

\begin{tabular}{|c|c|c|c|c|c|c|c|}
\hline \multicolumn{2}{|c|}{ Parameters } & Control $(n=30)$ & $\begin{array}{c}\mathrm{DM}+\mathrm{CAD} \\
(\mathrm{n}=30)\end{array}$ & $\operatorname{DM}(n=30)$ & $\square^{2}$-value & Df & $\begin{array}{c}\mathrm{P}- \\
\text { value }\end{array}$ \\
\hline \multirow[t]{2}{*}{ Sex } & Female & $12(40.0)$ & $13(43.3)$ & $10(33.3)$ & \multirow{2}{*}{1.425} & \multirow{2}{*}{2} & \multirow{2}{*}{0.490} \\
\hline & Male & $18(60.0)$ & $17(56.7)$ & $20(66.7)$ & & & \\
\hline \multirow[t]{3}{*}{ Religion } & Christian & $7(23.3)$ & $7(23.3)$ & $9(30)$ & \multirow{3}{*}{1.134} & \multirow{3}{*}{4} & \multirow{3}{*}{0.889} \\
\hline & Hindu & $22(73.3)$ & $22(73.3)$ & $19(63.3)$ & & & \\
\hline & Muslim & $1(3.33)$ & $1(3.33)$ & $2(6.67)$ & & & \\
\hline \multirow[t]{2}{*}{ Inhabitance } & Rural & $10(33.3)$ & $7(23.3)$ & $12(40)$ & \multirow[b]{2}{*}{1.933} & \multirow[b]{2}{*}{2} & \multirow[b]{2}{*}{0.380} \\
\hline & Urban & $20(66.7)$ & $23(76.7)$ & $18(60)$ & & & \\
\hline \multirow{2}{*}{$\begin{array}{c}\text { Educational } \\
\text { level }\end{array}$} & Illiterate & - & $2(6.67)$ & $3(10)$ & \multirow[b]{2}{*}{2.965} & \multirow[b]{2}{*}{2} & \multirow[b]{2}{*}{0.227} \\
\hline & Literate & $30(100)$ & $28(93.3)$ & $27(90)$ & & & \\
\hline \multirow[t]{2}{*}{ Working status } & Non sedentary & $7(23.3)$ & 14(46.7) & $16(53.3)$ & \multirow{2}{*}{6.150} & \multirow{2}{*}{2} & \multirow{2}{*}{0.046} \\
\hline & Sedentary & $23(76.7)$ & $16(53.3)$ & $14(46.7)$ & & & \\
\hline \multirow[t]{3}{*}{ EconomicStatus } & Up to Rs.5000 & $2(6.67)$ & - & $2(6.67)$ & & & \\
\hline & Rs.5000- 10000 & $23(76.7)$ & $28(93.3)$ & $28(93.3)$ & & & \\
\hline & Above Rs. 10000 & $5(16.7)$ & $2(6.67)$ & - & & & \\
\hline \multirow[t]{2}{*}{ Marital status } & Unmarried & $9(30.0)$ & - & - & & & \\
\hline & Married & $21(70.0)$ & $30(100)$ & $30(100)$ & & & \\
\hline
\end{tabular}

Table-1, shows the distribution of cases in terms of parameters over the three groups. Here, insignificant P-values for sex, religion, inhabitance, educational level and socio-economic status imply that these based line characteristics are comparable over the groups despite some visible variations pertaining to within the groups as well as between sub classifications of each parameter. These observed variations might be noticed due to chance factors and therefore they may be neglected. Henceforth, these basic characteristics (background variables) do not influence the main findings of the present study. 
Table-2. Group-wise distribution of cases according toBMI and hyperlipidemia:

* Percentage is shown in parenthesis

\begin{tabular}{|c|c|c|c|c|}
\hline \multirow[b]{2}{*}{ Parameters } & \multicolumn{3}{|c|}{ Group } & \\
\hline & Control $(n=30)$ & $\mathrm{DM}+\mathrm{CAD}(\mathrm{n}=30)$ & $\mathrm{DM}(\mathrm{n}=30)$ & \\
\hline \multirow[t]{2}{*}{ BMI kg/m2 } & Moderate & $2(6.7)$ & $9(30.0)$ & $13(43.3)$ \\
\hline & Normal & $28(93.3)$ & $21(70.0)$ & $17(56.7)$ \\
\hline \multirow[t]{2}{*}{ Hyperlipidemia } & No & $29(96.7)$ & $11(36.7)$ & $8(26.7)$ \\
\hline & Yes & $1(3.3)$ & $19(63.3)$ & $22(73.3)$ \\
\hline
\end{tabular}

Table-2, shows that 2 in the control, 13 in the DM and 9 in the DM+CAD group had moderate BMI respectively. But 28 in the control, 21 in the DM, and 17 in the DM + CAD group had normal BMI respectively. It was also observed that 1 in the control, 22 in the DM and 19 in the DM+CAD group had hyperlipidemia respectively.

Table-3. Group-wise distribution of Mean \pm SD of creatinine and Cystatin C:

\begin{tabular}{|c|c|c|c|c|}
\hline Parameters & & Mean \pm SD & F-value & P-value \\
\hline \multirow{4}{*}{ Creatinine $(\mathrm{mg} / \mathrm{dl})$} & Control $(\mathrm{n}=30)$ & $0.91 \pm 0.17$ & & \\
\hline & $\mathrm{DM}(\mathrm{n}=30)$ & $1.0 \pm 0.16$ & & \\
\hline & $\mathrm{DM}+\mathrm{CAD}(\mathrm{n}=30)$ & $0.97 \pm 0.17$ & 8.527 & $<0.001$ \\
\hline & Total $(n=90)$ & $0.99 \pm 0.18$ & & \\
\hline \multirow[t]{4}{*}{ Cystatin C(mg/l) } & Control $(\mathrm{n}=30)$ & $0.74 \pm 0.15$ & & \\
\hline & $\mathrm{DM}(\mathrm{n}=30)$ & $1.0 \pm 0.27$ & & \\
\hline & $\mathrm{DM}+\mathrm{CAD}$ & $0.93 \pm 0.24$ & 10.552 & $<0.001$ \\
\hline & Total $(n=90)$ & $0.89 \pm 0.25$ & & \\
\hline
\end{tabular}

Table-3, shows serum creatinine level of 0.91 for Control group which is followed by $\mathrm{DM}+\mathrm{CAD}(0.97)$ and highest in the group of DM (1.0). The difference is again tested by F-test and found to be a significant $(\mathrm{P}<$ $0.001)$; it implies that the creatinine level is significantly varied among Control, DM and DM+CADgroups. Similarly, a significant difference of mean cystatin $\mathrm{C}$ is recorded $(\mathrm{P}<0.001)$ among the three groups, being indicating DM group is the highest with 1.0 while DM+CAD groupwith 0.93 is the second highest, and the lowest in Control with 0.74 . As such, as per the finding, one may conclude that cystatin $\mathrm{C}$ differs significantly from Control group as well as from DM group to DM+CAD group.

Correlation matrix analysis of risk creatinine with cystatin $\mathrm{C}$ with other different clinical findings viz. SBP, DBP, age, HbAlc and lipid profiles showed that creatinine is said to be significantly correlated with cystatin $\mathrm{C}$, age and HBA1c .

Table-5. Distribution of Quartiles according to serum level of Cystatin C and HbA1c

Table-5, shows first, second and third quartiles with association between CsTc and HbA1c.

\begin{tabular}{|c|c|c|c|}
\hline & $\mathrm{Cr}(\mathrm{mg} / \mathrm{dl})$ & $\mathrm{CsTc}(\mathrm{mg} / \mathrm{l})$ & $\mathrm{HbAlc}(\%)$ \\
\hline First quartile & 0.860 & 0.700 & 5.60 \\
\hline Second quartile & 0.980 & 0.900 & 7.55 \\
\hline Third quartile & 1.165 & 0.985 & 8.80 \\
\hline
\end{tabular}

Table-6..Correlation matrix of risk factors and Cystatin C

\begin{tabular}{|c|c|}
\hline & CsTc $(\mathrm{mg} / \mathrm{l})$ \\
\hline $\operatorname{Cr}(\mathrm{mg} / \mathrm{dl})$ & $0.417(* *)$ \\
\hline Age(yrs) & $0.350(* *)$ \\
\hline & \\
\hline
\end{tabular}




\begin{tabular}{|c|c|}
\hline $\mathrm{SBP}(\mathrm{mmHg})$ & $0.296(* *)$ \\
\hline $\mathrm{DBP}(\mathrm{mmHg})$ & $0.331(* *)$ \\
\hline $\mathrm{HbAlc}(\%)$ & $0.286(* *)$ \\
\hline $\mathrm{TC}(\mathrm{mg} / \mathrm{dl})$ & $0.300(* *)$ \\
\hline LDL-C (mg/dl) & $0.270(* *)$ \\
\hline HDL-C (mg/dl) & $-0.271(* *)$ \\
\hline $\mathrm{TG}(\mathrm{mg} / \mathrm{dl})$ & 0.131 \\
\hline
\end{tabular}

It may be observed from the table- 6 that there is a strong positive correlation between Creatinine and Cystatin $\mathrm{C}(\mathrm{r}=0.417)$. Again, age of the patient has a significant impact on the serum level of creatinine as evident by $\mathrm{r}=0.221$. On the contrary, blood pressure $(\mathrm{mmHg})$ in both systolic and diastolic does not have significant relation with the serum, but they have positive association with the serum. Nonetheless, HbA1c has a highly significant impact on the serum, one of the markers of CAD. This statement is supported by $\mathrm{r}=0.292$, which is very much significant even at $1 \%$ probability level. None of the lipid profile has significant relation with the serum statistically. But except HDL-C there is a direct association of them with the serum.

It is worthwhile to mention here that all the risk factors considered except the TG have highly significant influence on the CsTc as evident by all the corresponding P-values of less than 0.001. Nevertheless, HDL-C has significant correlation impact on the serum.

Table-7. Results of Receiver Operating Characteristic (ROC) analysis of CsTc on predicting CAD

\begin{tabular}{|c|c|c|c|c|c|}
\hline Parameter & Area under curve (95\% CI) & P-value & Cut-off value & Sensitivity & Specificity \\
\hline Cystatin C & $0.795(0.699-0.891)$ & $<.001$ & 0.8 & 0.816 & 0.633 \\
\hline
\end{tabular}

From table-7, it was observed that from the area under the ROC curve which was found to be 0.795 (graphically also shown in fig-1). It indirectly indicates that diagnostic accuracy of CsTc to detect CAD was $79.5 \%$. The Receiver Operating Characteristic (ROC) analysis was performed to determine CsTc level as predictor of $\mathrm{CAD}$, in terms of its sensitivity and specificity of the serum level. The cut off value was derived on the basis of the value, which gave the maximum sensitivity and specificity with minimum variation between them. From the table-14, the cutoff point of $\geq 0.8 \mathrm{mg} / 1$ serum CsTc level was recorded with $81.6 \%$ sensitivity and $63.3 \%$ specificity respectively

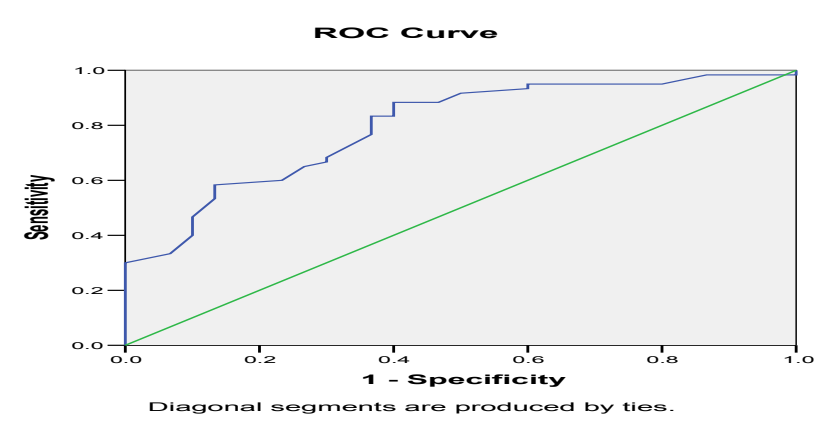

Fig-1: Showing ROC curve along with sensitivity and specificity of CsTe

\section{Discussion}

In the present study, the level of lipid profiles: TC, LDL-C, HDL-C and TG were significantly increased among all the cases irrespective of age and sex as compared to the control group. The mean serum level as mg/dl was TC (184.93), LDL-C (120.93), HDL-C (30.80), TG (138.33) \& TC (208.93), LDL-C (143.13), HDL-C (24.53), TG (152.00) in DM and DM+CAD group respectively. The mean serum creatinine level $(\mathrm{mg} / \mathrm{dl})$ were (1.0) in DM group and (0.97) in DM+CAD group respectively. The mean serum level of $\mathrm{HbA1c}(\%)$ were (7.96) and (8.79) in DM and DM+CAD group respectively. The mean serum Cystatin C level $(\mathrm{mg} / \mathrm{l})$ were $(1.0)$ and $(0.97)$ in $\mathrm{DM}$ and $\mathrm{DM}+\mathrm{CAD}$ group respectively.

The report of this study is similar to a study done at Islamic University-Gaza, Deanery who reported a 
mean serum level as $\mathrm{mg} / \mathrm{dl}$ of TC (186.99), LDL-C (124.82), and TG (140.68) respectively. The mean serum creatinine level as $\mathrm{mg} / \mathrm{dl}$ were (1.08) in DM group and (0.97) in DM+CAD group. The mean serum level of $\mathrm{HbAlc}$ were $(8.18 \%)$ and $(9.78 \%)$ in $\mathrm{DM}$ and $\mathrm{DM}+\mathrm{CAD}$ group the respectively, which was much higher than that of the present study. This might be due to difference in the race, sex and sociodemgraphic profile. ${ }^{[19]}$

The serum levels of the serum cystatin $\mathrm{C}$ were also significantly elevated among both the DM and $\mathrm{DM}+\mathrm{CAD}$ group of patients when compared with controls. The serum level of serum CsTc as $\mathrm{mg} / 1$ were found to be (1.1) and (0.97) in DM and DM+CAD group respectively while serum creatinine level as $\mathrm{mg} / \mathrm{dl}$ were found to be (0.92) in DM group and (1.01) in DM+CAD group, while serum level of HbAlc were found to be (5.11\%) and (7.97\%). Among DM group the serum level as mg/dl of TC (187.99), LDL-C (125.82), HDL-C

(28.44) and TG (130.16), where as in CAD+DM group serum level of TC, LDL-C, HDL-C and TG as $\mathrm{mg} / \mathrm{dl}$ were $188.01,130.11,20.64$, and 136.98 respectively.

Another study done by Goerge Ket $\boldsymbol{a l}^{[20]}$ reported a mean serum level of $0.99 \mathrm{mg} / 1$ for serum CsTc among DM+CAD group of patients which was much higher than the finding of present study. In the present study, it is found that DM +CAD group patients had a higher serum level of, HbAlc, TC, LDL-C, HDL-C and TG as compared to the DM+CAD group patients. However DM+CAD group had lower CsTc level than DM group. A level of $0.91 \mathrm{mg} / 1,8.73 \%$ for $\mathrm{CsTc}$ and $\mathrm{HbAlc}$ respectively for $\mathrm{DM}+\mathrm{CAD}$ group of patients was reported by study done by Muntner $\mathbf{P}$ et $\boldsymbol{a l}^{[21]}$ which was just lower than the present study.

The increased levels of serum CsTc among the DM+CAD patients as compared to the controls indicate that there is a confirmed risk of CAD in DM patients. The difference in the serum level of CsTc among $\mathrm{DM}+\mathrm{CAD}$ group patients may be due to age, sex, and race and sociodemgraphic profile. The significance increased in the serum level of $\mathrm{HbAlc}$ among $\mathrm{DM}+\mathrm{CAD}$ group of patient as compared to the control group of patient is clearly indicates that there is a significant increase of blood sugar due to DM-2. The diagnostic utilityof serum CsTc test is best evaluated by different predictive value of quartiles of the test. Among the control, DM and DM+CAD patients, the overall mean value of the serum cystatin $\mathrm{C}$ as $\mathrm{mg} / \mathrm{l}$ were $0.99,0.98$ and $1.0 \mathrm{mg} / \mathrm{l}$ respectively as reported by Finney Het al. ${ }^{[22]}$

In the present study CsTc is compared with creatinine and HbAlc. The first quartile of CsTc corresponds to a better glycaemic control with $\mathrm{HbA} 1 \mathrm{c} 5.6 \%$. In the present study it is noted that age, sex, smoking habits, drinking alcohol, literacy, working status and socioeconomic status has significant impact on the prevalence of CAD and CAD $+\mathrm{DM}$. Certain non-renal mechanisms have been proposed by Mussap M $\boldsymbol{e t}$ $\boldsymbol{a} \boldsymbol{l}^{[18]}$ to explain CsTc's association with CAD risk - either direct pathologic effects or associations with other risk factors. CsTc might reflect an increased inflammatory state that contributes to atherosclerotic plaque vulnerability and a higher risk of plaque rupture and thrombotic complications.

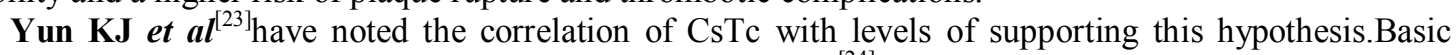
investigations demonstrate those thrombi proposed by Karin $\mathbf{A}$ et $\boldsymbol{a l}^{[24]}$ formations are related to the level of

collagen in the atheroma's cap. This suggests that CsTc expression may induce degradation of the collagen forming cathepsins. Horng HC et $\boldsymbol{a l}^{\left[{ }^{25]}\right.}$ reported that, an intriguing interaction between CsTc and cardio-preventive medications exists for predicting inducible ischemia. In stratified analyses, it was observed that there was strongest association between CsTc and ischemia among participants who were not using betablockers and statins. As noted by Grubb A et al ${ }^{[26]}$ pharmacologic therapies are known to inhibit atherogenesis and lead to regression in plaque development. Plaque stabilization could potentially explain why CsTc might be a less sensitive marker for inducible ischemia among patients treated with these therapies.

Cystatin C has a direct effect on atherosclerosis and inflammation as per study of Dharnidharka VR et al. ${ }^{[27]} \mathrm{CsTc}$ is the most potent endogenous inhibitor of cysteine proteinases such as cathepsins, elastase and papain. Because cysteine proteinases have a prominent role in protein catabolism and the cleavage of membrane and extracellular matrix proteins, CsTc probably has a central regulatory function in the extracellular compartment.Consequently, imbalance between the proteases and inhibitors may affect the cardiovascular system. In addition, CsTc is probably involved in the regulation of inflammatory processes, where it inhibits polymorphonuclear cell chemotaxis and phagocytosis as per Lee $\mathbf{M}$ et $\boldsymbol{~ a l}^{[28]}$

In the present study, it was demonstrated that among persons with CsTc concentrations in the highest quartile of our cross-sectional study, HbAlc had higher values $(8.8 \%)$ and creatinine values $>1.65 \mathrm{mg} / \mathrm{dl}$.The Receiver Operating Characteristic (ROC) analysis is performed to determine CsTc level as predictor of CAD, in terms of its sensitivity and specificity of the serum level. The cut off value is derived on the basis of the value, which gives the maximum sensitivity and specificity with minimum variation between them.

These results demonstrated that the risk of adverse events attributable to kidney disease was not completely captured by estimates of kidney function routinely used in clinical practice. More accurate characterization of kidney function in persons with CAD could allow targeted therapies that might improve prognosis and limit use of tests or procedures that have high risk for acute renal failure and associated consequences.Studies done by Poggio ED et $\boldsymbol{a l}^{[29]}$ demonstrated that CsTc could predict all-cause mortality, cardiovascular events, and incident CHF. Among elderly ambulatory cohorts predominantly without CAD. The 
predictive value of CsTc among outpatients with prevalent CAD had not been studied extensively.

\section{Conclusion}

The study have shown a positive and strong evidence of higher mean serum CsTc levels in patients of type 2 diabetes mellitus with coronary artery disease suggesting that total serum CsTc level is one of the risk factors predisposing to coronary artery disease. Total serum CsTc levels are found to be higher in male both in patients of type 2 diabetes mellitus with and without coronary artery disease as well as in controls compared to their respective female counterparts. Thus, a serum CsTc level finds a link an endothelial dysfunction marker and the development of coronary artery disease.

In addition, the study also shows that serum CsTc level is significantly correlated positively with LDL$\mathrm{C}$ and negatively correlated with HDL-C. There is also positive correlation of serum CsTc level with glycosylated hemoglobin in DM-2 with CAD implicating a certain relationship of vascular complications like $\mathrm{MI}$, angina and IHD, which are related with accelerated atherosclerosis.A significant difference of mean CsTc has been recorded among the three groups, indicating DM the highest while, DM+CADnext to the highest, and the lowest to Control. Moreover, higher CsTc concentrations can predict increased risk of these adverse clinical outcomes even among persons without derangement of kidney function. However, future prospective studies of serum CsTc measurement will be useful for the prediction of cardiovascular events among persons with coronary artery disease.

\section{References}

[1] Park K. Epidemiology of Chronic Non-Communicable diseases \& Conditions. In: Text book of Preventive and Social Medicine. $18^{\text {th }}$ ed. Jabalpur: M/S Banarsidas Bhanot; 2005. 290.

[2] Park K. Epidemiology of Chronic Non-Communicable diseases \& conditions. In: Text book of Prevention and Social Medicine. $18^{\text {th }}$ ed. Jabalpur: M/S Banarsidas Bhanot; 2005.288.

[3] Tanil H, Psaty BM, Newman AB, Siscovick D, Shlipak MG, Apregård J et al, 1961: Discovery of Cytatin C The measurement of serum cystatin C-concentration as a simple measure of kidney function is rapidly gaining in popularity. Karolinska University Hospital, Stockholm; Atherosclerosis, 199(2008):397.

[4] Park K. Epidemiology of Chronic Non-Communicable diseases \& conditions. In: Text book of Prevention and Social Medicine. $18^{\text {th }}$ ed. Jabalpur: M/S Banarsidas Bhanot; 2005.287.

[5] Harrison's Principle of Internal Medicine. $17^{\text {th }}$ ed. New York: Mc Graw Hill Medical; 2008(1).270-4.

[6] Poole W, King W III, Roberts, Prvstowsky N. Cardiovascular diseases. In: Fuster V, Specner B. Hurts's the Heart. $12^{\text {th }}$ ed. New York: Mc GrawHill Medical; 2007 (1). 2080.

[7] Seliger SL, Randers E, Erlandsen EJ, Fan D, McCulloch CE, McCulloch CE et al. Serum cystatin C as an endogenous marker of renal function: a review. Clin Chem Lab Med. 1999; 37: 389-395.

[8] Itoh Y, Eun Hee Koh,Woo Je Lee, Min-Seon Kim,Woo Je Lee and Min-Seon Kim.Biological Function of Cystatin C and its role:NEJM 2004; 351(13): 1296-305.

[9] Eun Hee Cho and Eun Hee Koh. Cystatin C Super family and their Subclasses. Diabetes Annual 1994: 193-212.

[10] Weiner, DE, Walker LC et al. Chronic Kidney Disease as a Risk Factor for Cardiovascular Disease and All-Cause Mortality: J Am Soc Nephrol 2004; 15: 1307-15.

[11] Selvin K, Michael G. Shlipak, Levey AS, Berg RL, Hall PM, Johansson L et al. The most promising use of cystatin C will be as a marker for preclinical or early kidney disease, among persons with creatinine-based eGFR in the 'normal range' but elevated cystatin C. American Journal of Kidney Diseases,51(2008):358.

[12] Kiuchi J, Robert G, Hossain MA, Emara M, EL Moselhi H, Shoker A et al. Comparing measures of cystatin C in human sera by three methods. Am J Nephrol. 2009; 29 (5): 381-91.

[13] Goldstein HE, Little RR, Widdmayer HM, 1994: Estimation of Glycosylated Hemoglobin (HbA1c).TIETZ Clinical Chemistry $6^{\text {th }}$ Ed.

[14] Allain C C, Pon L S, Chan S G, Richard W, Paul F Fu. Enzymatic determination of Total Serum Cholesterol. Clin Chem 1974;20(4):470-75.

[15] ucole G, Harold D. Quantitative Determination of Serum Triglycerides by the use of Enzymes. Clin Chem 1973;19(5):476-81.

[16] Steele B W, Kochler D F, Azar M M, Blas Z, Kpwaski P T. High density lipoprotein fractions, prepared by a precipitation technique. Clin Chem 1976; 22(1):98-01.

[17] Fredrickson D S, Fried W T, Dey R I. Estimation of the concentration of low density cholesterol in plasma, without use of the preparative ultracentrifuge. Clin Chem 1972;18:499-04.

[18] BioVendor Human Cystatin C ELISA: Estimation of Human cystatin C by kit method. Version: 2129090919.

[19] Abed EL, Rahman Abu Hilal, Mohammad Shubair, Chiarelli F, Verrotti A, Mohn A et al. Early Markers for Diabetic Nephropathy in Urine of Type 2Diabetics in Southern Gaza Strip, Islamic University-Gaza. Ann Med 1997 ;29 (5): 439-445.

[20] Geroge K, Kpwaski P T, Yoshida Y, Kawamura T, Ikoma M, Fogo A et al. Effects of antihypertensive drugs on glomerular morphology with coronary artery disease. Kidney Int 1989; 36: 626-35.

[21] Muntner P, David M, Maahs, Lorraine G. Ogden, Adam Kretowski, Janet K. Snell-Bergeon et al. Low muscle mass and poor nutrition, serum creatinine may remain in the normal 'blind' range despite decreasing GFR; Heart and Soul Study American Journal of Kidney Diseases 2007; 49:365.

[22] Finney H, Chertow GM, Fan D, McCulloch CE, McCulloch CE, Hsu CYet al. Chronic kidney disease and the risk of death, cardiovascular events, and hospitalisation. N Engl J Med. 2004; 351:1296-9.

[23] Mussap M, Lars Wallentin, Won Gu Kim, Seung Hun Lee, Goldsmith DJ, Cho S et al. . Serum cystatin C as a new marker for noninvasive estimation of glomerular filtration rate and as a marker for early renal impairment Parikh Section of Nephrology; Clinical Journal of the American Society of Nephrology, 3(2008):1895.

[24] Yun KJ, Chow J, Lee M, Saver JL, Huang WH, Chang KH et al. Impact of elevated cystatin C level on cardiovascular disease risk in predominantly high cardiovascular risk populations: a meta-analysis. Circ Cardiovascular Qual, Outcomes 2010, 3:675-683.

[25] Karin A,Mares J, Laakso M, Eun Hee Kim, Ji Hee Yu, Sang Ah Lee et al. Glycemic control and the risk for coronary heart disease in patients with non-insulin-dependent diabetes. Acta Biochim. Pol. 2009; 56: 3: 455-463. 
[26] Horng H C, Sang Ah Lee, Eui Young Kim, Won Gu Kim, Seung Hun Lee, Eun Hee Cho et al. Serum cystatin C as a biomarker for predicting coronary artery disease in diabetes. Korean Diabetes J. 2010 Apr; 34(2): 84-5. Epub 2010 Apr 30.

[27] Grubb A ,Janowski R, Kozak M, Abrahamson M, Jaskolski M, Eui Young Kim. 3D Domain- swapped human cystain C with amyloidlike intermolecular $\beta$ - sheets. Proteins: Structure, Function, and Bioinformatics. 2005; 61: 570- 578.

[28] Dharnidharka VR, Eun Hee Kim, Ji Hee Yu, Sang Ah Lee, Eui Young Kim, Won Gu Kim et al. Comparative assessment of cystatin C and creatinine for determining renal function in diabetic with CAD, Indian J Neprol 2005; 15: $91-4$.

[29] Lee M , Mevlut Koc, Mustafa Kemal Batur, Osman Karaarslan, Gülcan Abali, Wild PS et al.Cathepsin Enzymes and Cystatin C, Circulation. 2004; 110:2342-2348. 